\title{
A Coronavirus Detected in the Vampire Bat Desmodus rotundus
}

\author{
Paulo Eduardo Brandão ${ }^{1,2}$, Karin Scheffer ${ }^{3,2}$, Laura Yaneth Villarreal ${ }^{4,2}$, Samira Achkar ${ }^{3}$, Rafael de Novaes Oliveira ${ }^{3,2}$, Willian de \\ Oliveira Fahl ${ }^{3,2}$, Juliana Galera Castilho ${ }^{2,3}$, Ivanete Kotait ${ }^{3,2}$ and Leonardo José Richtzenhain ${ }^{1,2}$ \\ ${ }^{1}$ Department of Preventive Veterinary Medicine and Animal Health, College of Veterinary Medicine, University of São Paulo; ${ }^{2}$ Coronavirus \\ Research Group; ${ }^{3}$ Pasteur Institute; ${ }^{4}$ Intervet-Schering Plough; São Paulo, SP, Brazil
}

\begin{abstract}
This article reports on the identification of a group 2 coronavirus (BatCoV DR/2007) in a Desmodus rotundus vampire bat in Brazil. Phylogenetic analysis of ORF1b revealed that BatCoV DR/2007 originates from a unique lineage in the archetypical group 2 coronaviruses, as described for bat species elsewhere with putative importance in Public Health.

Key-Words: Coronavirus, vampire bat, RNA-dependent RNA-polymerase.
\end{abstract}

Coronaviruses (Coronavirus: Coronaviridae) have long been associated with a diverse set of diseases in a wide range of mammal an avian hosts, including humans, in which a new species in the genus named HCoV-SARS has been implicated in the etiology of severe acute respiratory syndrome [1].

Virions are enveloped and highly pleomorphic, with 50 to $200 \mathrm{~nm}$ in diameter, and a positive-sense ssRNA as genome, in which the 5' two-thrids (ORFs 1a and 1b) code for the replicase complex, highly conserved among members in each of the 3 groups the genus is divided, while the 3 ' final third codes for the structural proteins [2].

In bats, the first description of a coronavirus occurred in 2005 [3], when a group 1 coronavirus was found in Miniopterus spp. insectivorous bats in Hong Kong. Since then, groups 1 and 2 coronaviruses have been identified in a large number of insectivorous bat species in China, leading to the striking conclusion that the original host of the SARS coronavirus was a bat $[4,5]$.

Recently, the first bat coronavirus in the American Continent, named Rocky Mountain Bat Coronavirus, was detected in Eptesicus fuscus and Myotis occultus insectivorous bats in the USA [6], but hitherto no coronavirus has been reported in non-insectivorous bats.

This article reports a survey for coronaviruses in insectivorous and vampire bats from Brazil and the identification of a group 2 coronavirus in a vampire bat.

Specimens of the vampire bat $D$. rotundus $(\mathrm{n}=3)$, and 4 non-identified insectivorous bats were captured in São Paulo state, Southeastern Brazil, in 2005, as part of a continuous rabies surveillance program, when all of them where found rabies negative by routine rabies tests at Pasteur Institute, São Paulo, Brazil.

The complete enteric content of each of these bats was collected in a sterile microcentrifuge tube and prepared as

Received on 22 August 2008; revised 24 November 2008.

Address for correspondence: Dr. Paulo Eduardo Brandão, Department of Preventive Veterinary Medicine and Animal Health, College of Veterinary Medicine, University of São Paulo, Zip code: 05508-270, São Paulo, SP, Brazil. Phone number: 55-11-3091-7655. Fax: 55-113091-7928. E-mail address: paulo7926@usp.br.

The Brazilian Journal of Infectious Diseases 2008;12(6):466-468. (C) 2008 by The Brazilian Journal of Infectious Diseases and Contexto Publishing. All rights reserved.
20\% (v/v) suspensions in DEPC-treated water and clarified by centrifugation $\left(12,000 \mathrm{~g} / 30 \mathrm{~min} / 4^{\circ} \mathrm{C}\right)$.

Total RNA from the supernatant was extracted with TRIzol (Invitrogen ${ }^{\mathrm{TM}}$ ) and tested by a genus-specific RT-PCR targeted to amplify a 251-bp fragment of the ORF $1 \mathrm{~b}$ [8] and also to a group 2 coronavirus-specific nested-RT-PCR with a 136-bp amplicon internal to the first fragment with primers sense 5'TACTATGACTGGCAGAATGTTTCA3' and anti-sense 5'AACATCTTTAATAAGGCGRCGTAA 3' and M-MLV Reverse Transcriptase ${ }^{\mathrm{TM}}$ and Taq DNA Polymerase ${ }^{\mathrm{TM}}$ (Invitrogen, Carlsbad, CA, USA) as recommended by the manufacturer.

All seven samples resulted negative for the genus-specific RT-PCR; while the sample from the $D$. rotundus included in the study was the only that resulted in the 136-bp amplicon for the group 2 nested RT-PCR. This strain was named BatCoV DR/2007.

Next, the amplicon was purified from the agarose gel using Illustra ${ }^{\mathrm{TM}}$ DNA and Gel Band Purification Kit (GE Health Care, Buckinghamshire, UK) and submitted to bi-directional DNA sequencing with BigDye 3.1 (Applied Biosystems ${ }^{\mathrm{TM}}$ ) and the sequences where resolved in an ABI-377 Automatic DNA Sequencer (Applied Biosystems ${ }^{\mathrm{TM}}$ ). The sequence obtained was assigned GenBank Accession Number EU236685.

A Maximum Parsimony phylogenetic tree was built with the sequence from BatCoV DR/2007 and homologous sequences retrieved form the GenBank, corresponding to species in each of the 3 coronavirus groups (accescion numbers and strain names shown in Figure 1) and Bredavirus as an outgroup using MEGA 3 [8].

Strain BatCoV DR/2007 segregated in the cluster corresponding to the group 2 coronaviruses (Figure 1), depicting a unique lineage paraphyletic to the other subclusters in the group.

Mean nucleotide identities among BatCoV DR/2007 and other coronaviruses where $93.6 \%$ with archetypical group 2 coronaviruses (bovine coronavirus, human coronavirus OC43 , mouse hepatitis virus and rat syalodacryoadenitis virus), $82.3 \%$ with group 2 bat coronaviruses (BatHKUs), $72.5 \%$ with group 3, 69.9\% with group 1 and $66.6 \%$ with SARS coronavirus. All sequences included in the phylogenetic tree segregated in the expected groups of coronaviruses with the correct molecular tree topology described for the genus [9] and high 
Figure 1. Maximum parsimony rooted phylogenetic tree for a segment of the ORF1b of the Coronavirus genus, showing each of the three groups in the genus and in bold and underlined the strain BatCoV DR/2007 detected in the enteric content of a Desmodus rotundus bat. Numbers at each node are 1,000 replicates bootstrap values.

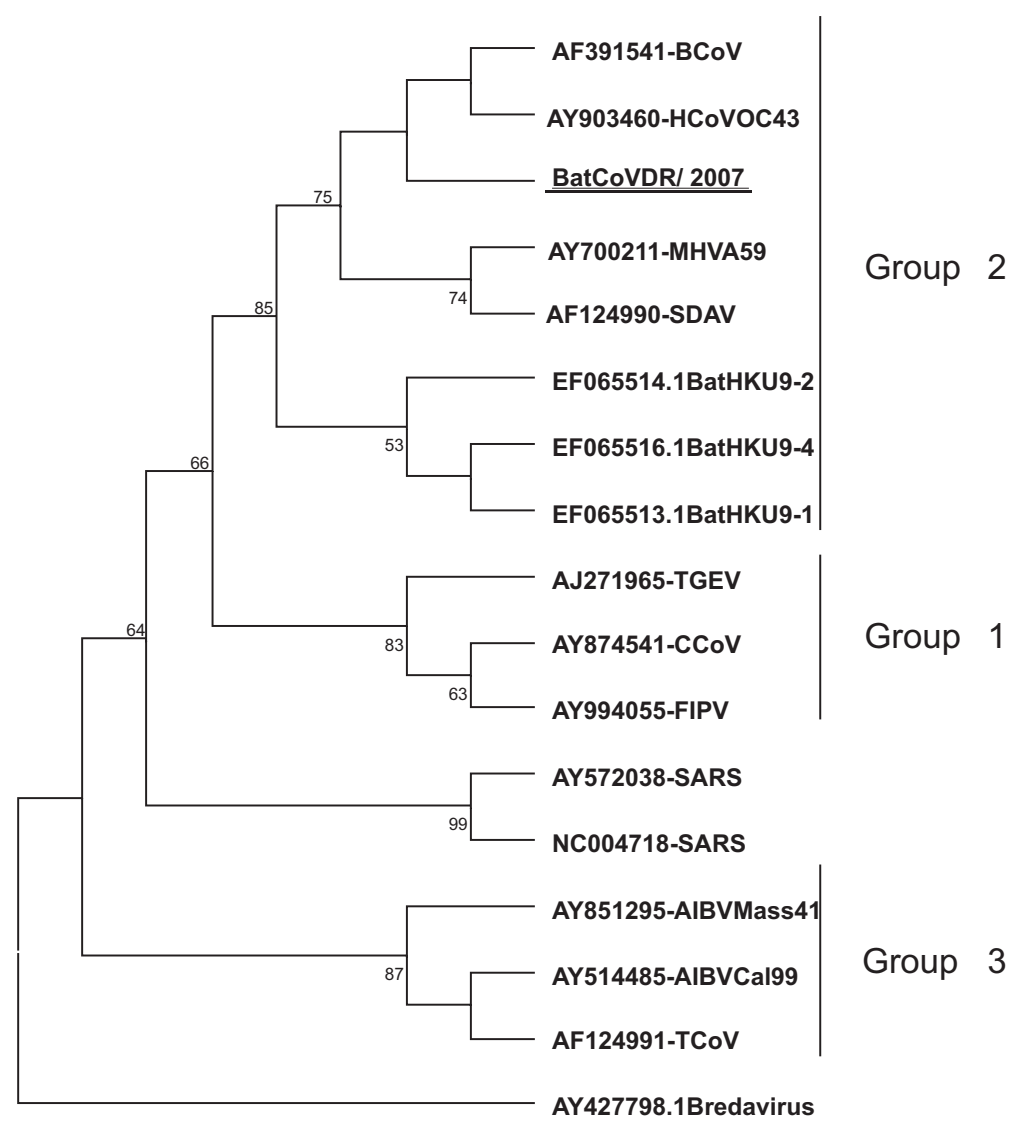

bootstrap values, what validates both the molecular marker and the sequence size chosen for typing.

Bat species thus far identified as reservoirs for coronaviruses were all found asymptomatic [6,10], still have not only been proposed as the original hosts for the SARS coronavirus [11] but also pointed as the hosts for an ancestor coronavirus that originated all coronavirus lineages currently known [10].

It is noteworthy that antibodies against coronaviruses such as SARS-like coronavirus were found widespread in bat populations in China, but a much lower frequency of coronavirus RNA was found in these same populations [12], pointing out that bats might be infected by coronaviruses but shed these at low titers [6], what could be then basis of the low frequency of coronavirus RNA recovery in the present research.

Desmodus rotundus poses a serious treat both for animal and human health in Latin America as this species is a major reservoir for rabies virus in this region [13], what has restricted investigations on the importance of these bats as potential carriers of other viral pathogens.

Initially, the finding of a coronavirus in the enteric content of a $D$. rotundus allow one to hypothesize that these bats may harbor a coronavirus species of yet unknown importance in public health.

As only the group 2-specific nested RT-PCR resulted positive for the $D$. rotundus sample, while the genus-specific, non-nested RT-PCR did not, one can infer that strain BatCoV DR/2007 was present at a low titer in the sample, supporting the theory of a highly stable host/ parasite relationship among coronaviruses and bats [10].

The coronavirus strain BatCoV DR/2007 described herein derives from a unique lineage among the other classic group 2 coronaviruses, but apart from other group 2 coronaviruses detected in bats such as the HKUs bat coronaviruses.

Nonetheless, an intense investigative effort has still to be carried out in order to gather more consistent sequence data for an elucidative classification of BatCoV DR/2007. Besides, a larger number of specimens of $D$. rotundus has yet to be sampled with the aim of making a conclusive connection between these and the group 2 coronavirus described herein.

As a conclusion, a coronavirus has been identified in the enteric content of a Desmodus rotundus vampire bat that can be classified, based on a small segment of the ORF1b, in the group 2 of the genus Coronavirus. 
Bat species other than the insectivorous ones, so far the only ones identified as reservoirs for coronaviruses in the Chiroptera, also may act as reservoirs of exclusive coronavirus lineages.

Finally, bringing these conclusions to the field of Public Health, the coronavirus strain described herein might be considered a putative pathogen for human diseases if a spillover event takes place, once bats, as already mentioned, are the origin of HCoV-SARS, making coronaviruses surveillance on bats and the increase of public awareness on battransmitted diseases relevant actions.

\section{References}

1. Ksiazek T.G., Erdman D., Goldsmith C.S., et al. A novel coronavirus associated with severe acute respiratory syndrome. N Engl J Med 2003;348:1953-66.

2. Masters P.S. The molecular biology of coronaviruses. Adv Virus Res 2006;66:193-292.

3. Poon L.L., Chu D.K., Chan K.H., et al. Identification of a novel coronavirus in bats. J Virol 2005;79:2001-9.

4. Tang X.C., Zhang J.X., Zhang S.Y., et al. Prevalence and genetic diversity of coronaviruses in bats from China. J Virol 2006;80:7481-90.
5. Wang L.F., Shi Z., Zhang S., et al. Review of bats and SARS. Emerg Infect Dis 2006;12:1834-40.

6. Dominguez S.R., O’Shea T.J., Oko L.M., Holmes K.V. Detection of group 1 coronaviruses in bats in North America. Emerg Infect Dis 2007; 13:1295-300.

7. Stephensen C.B., Casebolt D.B., Gangopadhyay N.N. Phylogenetic analysis of a highly conserved region of the polymerase gene from 11 coronaviruses and development of a consensus polymerase chain reaction assay. Virus Res 1999;60:181-9.

8. Kumar S., Tamura K., Nei M. MEGA3: Integrated Software for Molecular Evolutionary Genetics Analysis and Sequence Alignment. Brief. Bioinformatics 2004;5:150-63.

9. González J.M., Gomez-Puertas P., Cavanagh D., et al. Comparative sequence analysis to revise the current taxonomy of the family Coronaviridae. Arch Virol 2003;148:2207-35.

10. Vijaykrishna D., Smith G.J., Zhang J.X., et al. Evolutionary insights into the ecology of coronaviruses. J Virol 2007;81:4012-20.

11. Lau S.K., Woo P.C., Li K.S., et al. Severe acute respiratory syndrome coronavirus-like virus in Chinese horseshoe bats. Proc Natl Acad Sci U S A 2005;102:14040-5.

12. Shi Z., Hu Z. A review of studies on animal reservoirs of the SARS coronavirus.Virus Res 2007; Apr 20; [Epub ahead of print]. Available from http://www.sciencedirect.com.

13. da Rosa E.S., Kotait I., Barbosa T.F., et al.Bat-transmitted human rabies outbreaks, Brazilian Amazon. Emerg Infect Dis 2006;12:1197-202. 